\title{
Psychosocial and behavioral outcomes of genomic testing in cancer: a systematic review
}

\author{
Tatiane Yanes $\mathbb{C}^{1,2} \cdot$ Amanda M. Willis ${ }^{1} \cdot$ Bettina Meiser ${ }^{1} \cdot$ Katherine M. Tucker ${ }^{1,3} \cdot$ Megan Best $\mathbb{C}^{4,5}$
}

Received: 28 March 2018 / Revised: 26 July 2018 / Accepted: 9 August 2018 / Published online: 11 September 2018

(c) European Society of Human Genetics 2018

\begin{abstract}
Psychosocial and behavioral outcomes of genetic testing in oncology are well known, however, it is unclear how these findings will generalize to more complex genomic testing. The aim of this systematic review was to assess the psychosocial and behavioral outcomes of cancer genomic testing. Studies were selected for inclusion if they were published from January 2003 to January 2017 and addressed psychological and behavioral outcomes of cancer genomic testing in adults. A review of four databases identified 9620 abstracts, with 22 publications meeting the inclusion criteria. Of the included articles, 11 studies reported on outcomes of germline testing, with three articles assessing panel testing and eight SNP testing. No studies assessed the outcomes of WGS or WES. Eleven articles assessed the outcomes of somatic testing, including testing for cancer prognosis and for personalized therapies. Studies were biased toward breast cancer and Caucasian women with high education and socioeconomic status. While studies demonstrated limited adverse psychological outcomes associated with genomic testing, a lack of consistency in psychosocial measures precluded any meta-analysis. Changes in health behavior following positive results were limited, and in some cases risk perception was not altered following genomic testing. There is limited evidence of adverse psychosocial outcomes and changes in health behavior following genomic testing to assess cancer risk. Findings from this review highlight the need for longitudinal research with superior methodological and theoretical design.
\end{abstract}

\section{Introduction}

Improved understanding of the molecular basis of cancer has led to major advances in its management. Reduced costs and improved technology meant that genomic profiling is

Electronic supplementary material The online version of this article (https://doi.org/10.1038/s41431-018-0257-5) contains supplementary material, which is available to authorized users.

Tatiane Yanes

t.yanes@student.unsw.edu.au

1 Prince of Wales Clinical School, Faculty of Medicine, UNSW, Sydney, Australia

2 School of Psychiatry, Faculty of Medicine, UNSW, Sydney, Australia

3 Hereditary Cancer Clinic, Prince of Wales Hospital, Sydney, Australia

4 Psycho-oncology Co-operative Research Group (PoCoG), University of Sydney, Sydney, Australia

5 Faculty of Medicine, University of Sydney, Sydney, Australia increasingly used in the cancer setting. Genomic testing is used in several ways in the management of cancer including (i) somatic testing: tumor testing for assessment of prognosis, to guide treatment choices; and (ii) germline testing including whole-genome screening (WGS), whole-exome sequencing (WES), panel testing, and single nucleotide polymorphism (SNP) testing to identify cancer risk, guide surveillance, and preventative behavioral interventions.

Although most people undergoing genetic testing in the cancer setting appeared to cope well with testing results, research has suggested that the process was associated with several psychosocial problems in the first few months postreceipt of result [1]. These include an increase in cancerspecific distress and anxiety in carriers [2], and difficulties in aligning perceived risk with objective risk [3]. In terms of behavioral impact, genetic testing generally resulted in increased surveillance behaviors of both carriers and noncarriers, but more often in carriers [2]. In one study examining the risk-reducing surgery choices of women at high-risk for breast cancer, more chose risk-reducing surgery than breast surveillance. Furthermore, surgery was 
more common in women with higher perceived risk, but this perception was often incorrect [4].

It is unclear how these findings will generalize to more complex genomic testing. Genomic testing involves examination of multiple genes and in some cases, can represent an unprecedented volume of uncertain results to process. Given the rapid progress of genomic medicine, an understanding of the evidence, based on the psychological and behavioral impact of genomic testing, is urgently required to enable translation of discoveries into clinical outcomes in which individuals are adequately supported. It is only if people understand and act on their results that many of the potential benefits of genomic testing will be achieved. To the best of our knowledge, no systematic review of the literature in this area has been conducted, and hence we conducted a systematic review to investigate what is currently known about the psychosocial and behavioral impact of genomic testing.

\section{Materials and methods}

\section{Search strategy}

This systematic review was designed and conducted in accordance with the Preferred Reporting Items for Systematic Reviews and Meta-Analyses (PRISMA) guidelines [5]. A literature search of peer-reviewed publications was conducted using the MEDLINE, Embase, PsycINFO, and CINAHL databases. Search strings are outlined in the Supplementary Table I. The search was limited to articles in English, published from January 2003 to January 2017. The year 2003 was selected, as it coincides with the conclusion of the Human Genome Project [6]. Additional publications were identified from the reference list of accepted articles and correspondence from field experts.

\section{Selection criteria}

Quantitative and qualitative studies were eligible for inclusion if they were original research addressing psychological or behavioral outcomes of cancer genomic testing in adults. Genomic testing of germline and somatic cells were included. Studies assessing outcomes in pediatric settings, direct-to-consumer testing, non-cancer diagnosis, hypothetical scenarios, or healthcare professionals' views were excluded from this review, as were studies where participants responses were not reported separately. Three authors, TY, AW, and MB independently reviewed the abstracts and/or articles identified through the search strategy described above. Authors BM and KT verified the inclusion and exclusion of articles and any disagreements were reconciled by discussion.

\section{Quality assessment}

All studies selected for inclusion were evaluated using the QualSyst assessment tool [7]. Authors TY, AW, and MB independently evaluated a random selection of ten studies. Quality assessments were reviewed by all authors and disagreements were reconciled by discussion. The remaining articles were divided among TY, AW, and MB for final quality assessment. No studies were excluded based on quality assessment.

\section{Data extraction and analysis}

Data extracted included sample characteristics (e.g., testing technology, cancer type, and country of origin), study design, and outcomes. Given the broad definition of genomic testing, findings were initially compared within each testing technology (somatic, germline, and SNP testing). Differences between each testing technology were also assessed.

\section{Results}

We identified 11,137 studies and exported them to Endnote $\mathrm{X} 7$. After removing duplicates, records were screened by title/abstract. Seventy-eight full-text articles were assessed for eligibility, with 22 articles relating to 20 unique studies included in this review (Fig. 1). Eleven studies reported on outcomes of germline testing, including three articles assessing panel testing [8-10] and eight SNP testing (Table I). No studies assessed the outcomes of WGS or WES. Of the studies that returned the SNP result, five returned polygenic risk score [11-15], with the remaining studies reporting individual SNP result [16-18]. Eleven articles assessed the outcomes of somatic testing [19-29].

\section{Quality assessment}

Quality scores ranged from 55 to $95 \%$ for qualitative articles and 73 to $100 \%$ for quantitative (Supplementary Table II). Common issues with qualitative studies included lack of theoretical framework, inappropriate design to answer the study question, and no reflexive practice reported. Among quantitative studies, description of statistical analysis and justification of sample size were often lacking. No studies were excluded based on quality assessment.

\section{Germline testing: panel test}

Knowledge of patients undergoing germline testing increased significantly compared to baseline after both 
Fig. 1 PRISMA Flowchart. The 22 studies included a total of 2529 participants (Table I). Most participants were female $(71 \%)$ of Caucasian background (74\%). Somatic testing was most commonly assessed in the context of recurrence risk to guide breast cancer treatment. Germline testing was assessed across a range of scenarios including among individuals with a high cancer risk, as a follow-up test for those with uninformative genetic test result, or in the general population to guide population risk screening

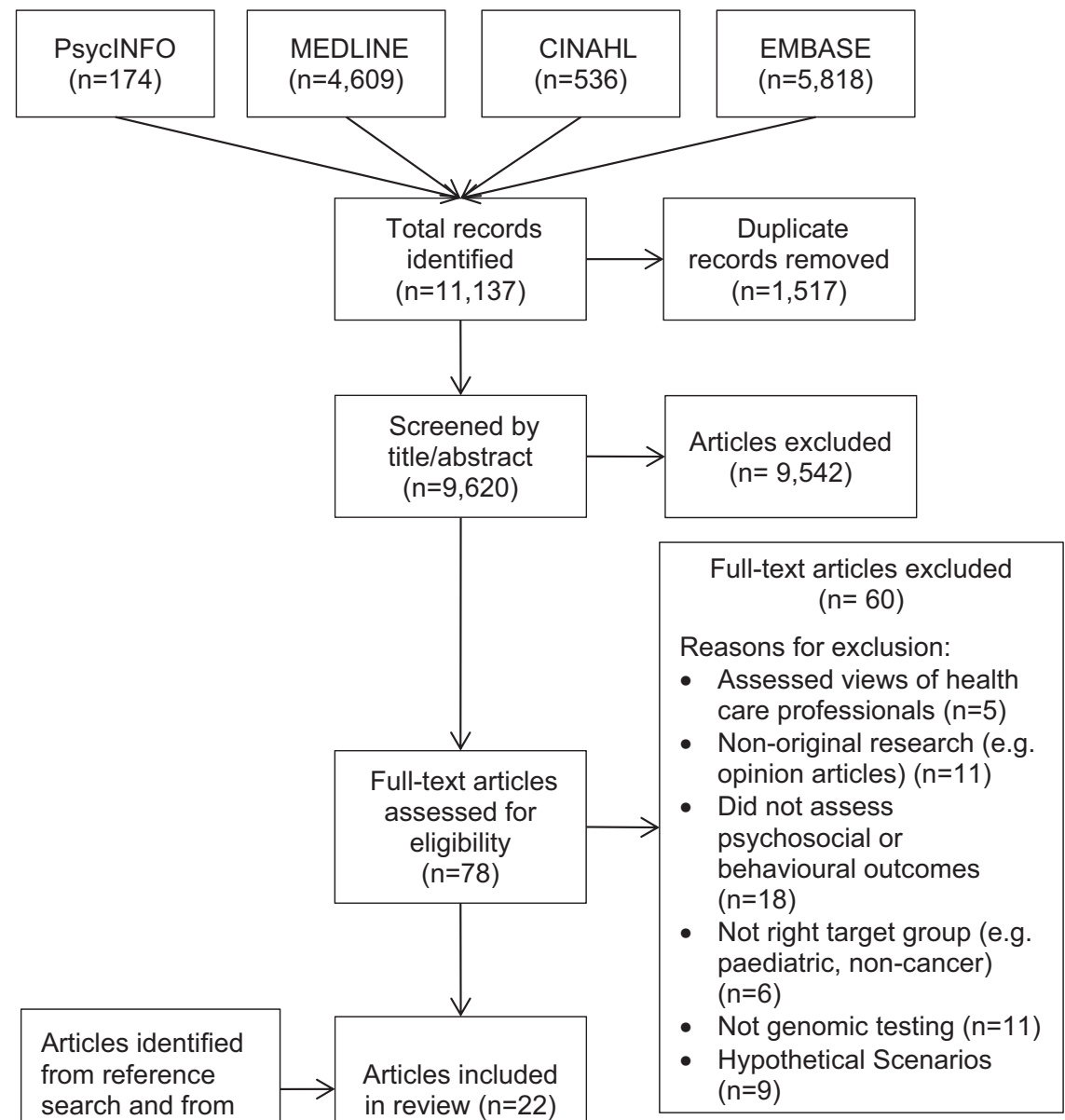

pre-test counseling and post-test counseling in one study, particularly knowledge of the limitations of testing (Supplementary Table III) [8]. Another study measuring knowledge about genetics reported that higher knowledge was associated with lower avoidance scores of event (where the event was receiving genomic results) and higher decisional satisfaction [9]. This study also reported some misunderstanding of genomic test results among participants, with variants of unknown significance (VUS) findings interpreted by participants as equivalent to a negative result, and only $80 \%$ of participants who received a positive result understood it to mean an increased risk of breast cancer [9]. No changes in perceptions of heredity or perceived risk of finding a hereditary cause for disease was reported between baseline and post-results [10]. Expectations of heredity were not associated with heredity-specific distress, coping style, or illness perception [10].

Two quantitative studies measured the aspects of general well-being, including generalized anxiety and depression. Both studies showed no change in general depression and anxiety, and cancer-specific avoidance and anxiety from baseline to follow-up $[8,10]$. One study comparing groups at a single time point reported higher distress among those in the no cancer/pathogenic variant group compared with other groups defined by cancer status and test results. This association was statistically significant when controlling for age, race, education, time since results, and knowledge [9]. Distress was higher among African-Americans compared with Caucasians and those with lower knowledge scores [9]. One study measuring illness perception found no statistically significant overall changes from baseline to follow-up at 2-3 weeks post-result, although those found to have a pathogenic variant reported more consequences and longer duration of their illness [10].

Two studies measured the impact of receiving genomic test results using the Multidimensional Impact of Cancer Risk Assessment (MICRA) [9, 10]. The MICRA scale was developed to assess the impact of genetic testing and consists of three subscales: test-related distress, positive experiences, and uncertainty [9, 10, 30]. One study administered an adapted version for hereditary risk across three time points and reported that distress scores remained low [10]. The other study measured differences between five groups defined by cancer status and variant status, 
Table I Characteristics of included studies $(n=22)$

\begin{tabular}{|c|c|c|}
\hline & $n$ & $\%$ \\
\hline \multicolumn{3}{|l|}{ Test type } \\
\hline Panel & 3 & 14 \\
\hline SNP & 3 & 14 \\
\hline Multiple SNPs (polygenic) & 5 & 22 \\
\hline Somatic & 11 & 50 \\
\hline \multicolumn{3}{|l|}{ Country of origin } \\
\hline USA & 10 & 45 \\
\hline Canada & 3 & 14 \\
\hline UK & 2 & 9 \\
\hline Australia & 3 & 14 \\
\hline France & 2 & 9 \\
\hline Netherlands & 2 & 9 \\
\hline \multicolumn{3}{|l|}{ Cohort* } \\
\hline $\begin{array}{l}\text { Undergoing germline genomic test and not previously tested } \\
\text { for heredity cancer }\end{array}$ & 3 & 14 \\
\hline Uninformative result from germline testing & 4 & 18 \\
\hline General population, unselected for disease status & 5 & 22 \\
\hline Undergoing cancer treatment & 11 & 50 \\
\hline \multicolumn{3}{|l|}{ Cancer Type* } \\
\hline Breast cancer & 15 & 68 \\
\hline Melanoma & 3 & 14 \\
\hline Prostate cancer & 3 & 14 \\
\hline Colorectal cancer & 4 & 18 \\
\hline Renal & 1 & 4 \\
\hline \multicolumn{3}{|l|}{ Study type } \\
\hline Qualitative & 6 & 27 \\
\hline Mixed-method & 2 & 9 \\
\hline Cross-sectional & 7 & 32 \\
\hline Longitudinal & 5 & 23 \\
\hline Pilot randomized control trial & 2 & 9 \\
\hline
\end{tabular}

*Value over $100 \%$, as some studies assessed more than one cancer type and populations group

namely: cancer/pathogenic variant positive, cancer/VUS, cancer/no pathogenic variant, no cancer/pathogenic variant positive, and no cancer/VUS [9]. Higher distress scores were reported among the no cancer/pathogenic variant positive group, followed by the no cancer/VUS group and cancer/pathogenic variant positive group [9]. Distress was also higher among the African-American cohort when compared with the Caucasian population. Test-related positive experience was lowest among the no cancer/VUS and the cancer/pathogenic variant positive group. Younger age was also associated with lower positive experience. There were no differences in uncertainty among the different groups assessed [9].

Uncertainty was measured in one study and did not change from pre- to post-testing [8]. Individuals' tolerance for ambiguity did not appear to impact on their coping, with one study finding no association between ambiguity tolerance and MICRA, avoidance and distress, or satisfaction scores [9].

After receiving the results of panel testing, participants in one study had only modest perceptions of the utility of testing, with overall perceived utility declining compared to pre-test scores. However, three of the five participants who received a positive result had increased perceived utility compared with pre-test [8]. These observed changes in perceived utility were not statistically significant, but the modest increase in perceived utility observed after receiving results in this study may reflect the fact that the medical management changed for only two participants as a result of the test results [8].

Decisional satisfaction and satisfaction with genetic services were high, did not change from pre-test counseling to post-results, and did not differ according to test results or cancer status [8-10]. However, satisfaction was lower for those with lower levels of education, lower knowledge, younger age, and Asian and Hispanic individuals (compared to Caucasians) [9]. Satisfaction with the decision to undergo genomic testing also decreased as the time since testing increased [9]. Few participants expressed regret, and dissatisfaction was mainly attributable to the long wait for results [10].

Participants appeared more likely to use their results in decision-making if they received a positive result, with those without a personal history of cancer taking up additional screening and those with a personal history of cancer using the results in treatment and/or surgical decisions [9]. Some participants who received a VUS or uninformative result reported that their results would influence their treatment or screening decisions [9]. Following receipt of the results, participants reported concerns about coping with the psychological burden of risk information, the impact on everyday life, and insurance and privacy concerns [9]. Few participants reported worrying about genetic discrimination, and even fewer had actually experienced discrimination based on their genomic test results [9].

\section{Germline testing: SNP}

When assessing the recall of results, most participants accurately recalled their SNP result up to a 5-month followup (Supplementary Table III) [11, 12]. Despite accurate recall, there is limited evidence that the SNP result altered the risk perception. Risk perception was often shaped by personal or family history of cancer [11, 12, 17]. For example, in one study of men with a family history of prostate cancer, heightened risk perceptions remained despite individual SNP result and counseling [12]. This was often explained by experience with prostate cancer in a close, relative, and fatalistic beliefs. 
Two qualitative studies reported emotional responses to SNP testing. Common responses included: reassurance, acceptance of result, and surprise or disappointment when the results did not match personal or family history of cancer. Participants also reported some skepticism given the small effect of individual SNPs [17, 18]. One study reported no changes to anxiety and distress post-results [18].

Psychological outcomes were assessed in three longitudinal studies. No significant changes in psychological measures between pre- and post-result (3-5 months) were reported, including no changes in cancer worry [12, 13, 17], no increased cancer-specific distress [12, 13, 17], and no changes to general distress and anxiety [12]. Furthermore, in a pilot randomized control trial, there was no evidence that skin cancer-related worry, psychological distress, and general well-being scores differed between the intervention group (those receiving polygenic result) and control group [13].

Sharing of personal SNP result with family members was often done due to perceived 'shared risk', or as a motivator for discussing risk and health behavior (Supplementary Table III) [14, 18]. Communication with healthcare professionals was influenced by risk result received. For example, in a pilot randomized trial, $41 \%$ of participants with a high SNP risk for melanoma had shared their results with healthcare professionals at 3-month follow-up compared with only $16 \%$ and $12 \%$ of the average and low-risk group, respectively [14].

There is limited evidence of change to health behavior (Supplementary Table III). Graves et al. [17] reported that $\sim 50 \%$ of their cohort made changes to diet and exercised 3 months post-receipt of the result. However, SNP result was not an independent predictor of behavior change. The author hypothesized that this behavior change was likely due to the education provided by the genetic counselor rather than the SNP result. In one study, men with family history of prostate cancer received SNP result for prostate cancer risk. Over one-third of participants reported feelings of reassurance after receiving their SNP result, however, none of the participants indicated that they would reduce their screening based on their SNP result [11]. In another study, melanoma prevention behavior at 3 months was greater in individuals who had both a family history and increased genetic risk [16]. In this study, behavior change was partially mediated by anxiety. Finally, in a pilot randomized control trial to date [13], investigators did not detect any significant change in risk-reducing or sun protection and screening behaviors at 3-month follow-up between the intervention and control groups. When stratified by risk groups (high, average, and low), a borderline significant reduction in intentional tanning was detected. The effect size was largest in the average risk group, followed by high-risk group and low-risk group.

\section{Somatic testing}

All but one study [23] exclusively involved patients with breast cancer, and most studies involved testing risk for recurrence (RFR) to guide treatment choices, with one study assessing the impact of biopsy testing to discover personalized treatment (Supplementary Table III) [23]. Knowledge about RFR somatic testing was generally found to be low [20, 21, 24, 26, 27]. However, most participants (76$95 \%$ ) undergoing these tests understood that the results could aid chemotherapy decisions [19, 21, 24, 26, 27, 29]. Fewer women (22-53\%) understood the relationship between the test result and chance of metastasis [21, 26]. The qualitative studies found that patient misunderstanding about RFR somatic testing led to increased anxiety [20, 24]. Patients with higher knowledge scores were more likely to have active roles in treatment decision-making, fewer concerns about testing, higher educational level, lower age, more recent cancer diagnosis, less comorbidity, higher income, or relatives who had previously undergone chemotherapy and were more likely to be in full-time employment, and Caucasian ancestry [21, 25-27]. Lipkus et al. [21] found that $62 \%$ of participants sought information about their results, and that those who sought information were significantly more knowledgeable. Perceived risk was significantly reduced after RFR somatic testing. Perceived RFR was also correlated with actual risk score of recurrence post-test [22, 29].

State anxiety for RFR testing decreased 12 months postresult [22]. State anxiety was positively correlated with decisional conflict both before and after testing [22]. Patient-reported factors that were associated with increased anxiety included: receiving results [29], a delay in return of results [19], or misconceptions about the meaning of the results [24]. Increased anxiety was associated with higher actual recurrence risk, more cancer-related distress, the genomic result not being available, and discordance between genomic result and recurrence score calculated through a standard clinical test [29]. Sulayman et al. [28] found that high levels of cancer-specific distress in $38.7 \%$ participants underwent testing for RFR. Overall, higher perceived RFR was associated with higher levels of worry, lower satisfaction, and Caucasian ancestry. Women who preferred a passive role in treatment decision-making and received an intermediate recurrence risk result had higher cancer-related distress, but not worry about recurrence [25, 28, 29].

Most participants (67-77\%) thought that the test results could be trusted [26, 27, 29] and that they were accurate $(71 \%)$ [26, 29] and useful $(76 \%)$ [26]. Reported benefits of test included the ability to reduce uncertainty [20] and provide better treatment options [20, 23, 24, 27, 29]. The latter result was shown in both testing for RFR and targeted 
therapy. Genomic testing gave participants a sense of empowerment [20], although overestimation of the validity of the tests was found to increase its value [20]. Reported drawbacks from testing included being given information about cancer that was better left unknown [29], and, in the case of search for personalized treatment, the risk of disappointment from non-findings or lack of access to relevant trial drugs [23]. However, Pellegrini et al. [24] found that those participants who did not qualify for a clinical trial rationalized their outcome, so they remained satisfied with the treatment. More concerns were reported among participants with higher perceived risk of recurrence [29].

Most participants $(96 \%)$ reported that they would have RFR testing again $[26,29]$ and $95 \%$ would recommend it to other women in the same situation [26, 29]. Lo et al. [22] found that decisional conflict reduced after RFR results were received. At 1-year post follow-up, $92.5 \%$ continued to feel satisfied that they had undergone testing and $95.5 \%$ were satisfied with their decision. Those patients not satisfied noted a negative impact on quality of life and self-image due to treatment. A second study also found a significant reduction in decisional conflict after the RFR results were reported [24].

Little attention was given to communication. Miller et al. [23] found that in a sample of patients with advanced cancer seeking new treatments, family obligations informed willingness to receive germline results following tumor testing, but results were perceived as burdensome and/or inconsequential, given their disease stage [23].

Somatic RFR results influenced chemotherapy decisions in several studies. Thirty-nine percent to $84 \%$ of participants reported that the results influenced adjuvant treatment decision-making (80.6\% after 12 months) [21, 22]. The decision to have chemotherapy differed according to level of risk [21, 29], but the most influential factor governing the treatment decision for some patients was the doctor $(55 \%)$ [21].

\section{Discussion}

This systematic review summarizes the current literature on the psychosocial and behavioral outcomes of cancer genomic testing. Traditionally, genetic testing in the oncology setting has been limited to testing of single genes associated with increased cancer risk. However, as demonstrated in this review, genomic testing is rapidly being integrated in several settings including for individuals with uninformative genetic test results, to guide population screening programs, and testing of tumor cells to guide cancer treatment. Furthermore, our review has identified that early research in this setting has primarily focused on females from Caucasian background with breast cancer. Hence a bias exists in the current literature and additional research in other cancer settings and demographic populations is needed.

The studies selected for inclusion vary widely in their aims, research design, methodology, and quality. There was no consistency in outcome measurement and often nonvalidated scales were used. Therefore, meta-analysis was not possible. Instead, a comparison of similar studies provided an initial insight and guidance for future research. Findings from this review highlight the need for further research on this topic with superior methodological and theoretical design. For example, including hereditary cancer-specific measures, consistent measures across studies, and prospective study designs.

Recently there has been movement away from measuring knowledge as an outcome of genetic testing [31]. Rather than conceptualizing knowledge as simply recalling facts, it is now understood to encompass many subjective and objective factors, such as relevance of information at the time of testing, risk perception, and how information has been provided [31]. Knowledge is also specific to each setting and individual disease, and thus knowledge measures are not easily transferable across settings [31]. These limitations in measuring knowledge were observed in this review, where no validated scales were used to assess knowledge, resulting in inconsistent findings of knowledge across studies. Despite these challenges, this review found positive outcomes associated with increased knowledge, including improved psychological well-being, more active role in treatment decision-making, fewer concerns about the test, and higher decisional satisfaction. Initiatives to improve patient knowledge before testing is therefore recommended.

Similarly to genetic testing [1], no evidence of long-term adverse psychological outcomes were identified in this review. However, only seven studies presented in this review had a longitudinal design, with the majority of follow-up being $<3$ months. Thus, there is limited research assessing the long-term psychological outcomes of cancer genomic testing. Additionally, most measures used to assess psychosocial outcomes were not validated or specific to hereditary cancer. For example, distress was often measured using the Impact of Event Scale, a scale originally developed to assess post-traumatic stress disorders [32]. Recently, there has been increasing debate over the lack of appropriate measures for psychosocial impact in genetic/ genomic medicine, with some authors highlighting a need to use cancer-specific scales such as the Psychosocial Aspects of Hereditary Cancer Questionnaire or MICRA to be used [1]. Previous studies have demonstrated that the MICRA has excellent discriminative validly, with BRCAl/ 2-positive patients having higher scores than those with informative BRCA1/2 results [30]. 
When assessing communication post-result, most participants were willing to share or had shared their results with family members. In some cases, sharing of results promoted a conversation about risk management and cancer prevention [14, 18]. Interestingly, when assessing SNP results, the level of risk received appeared to influence sharing of result, with those at high risk more likely to have done so when compared to those with low risk. Participants often reported the concept of 'shared risk' as a motivator for sharing results with family [14]. Similar findings have been reported with single-gene testing, with individuals more likely to share their results with family members if they received a positive result compared with uninformative or VUS results [33]. There are many reasons for this difference including a sense of responsibility toward relatives, a desire to prevent disease in relatives, and gain emotional support and advice [33]. This is a notable finding given that SNP-based risk is personalized and does not follow Mendelian inheritance, thus family risk cannot be inferred. Future studies should aim to explore people's understanding of SNP results and family communication.

As genomic testing is extended to the general population, it is expected that more people will have access to personalized risk management, which may include reduced screening [34]. Targeting screening to high-risk populations is likely to have significant benefits to health care as resources are allocated more efficiently and the burden of routine screening is reduced for those at the lowest risk. Previous studies with hypothetical designs have reported mixed responses to the proposed risk-based surveillance [35]. As well as initial support, participants reported some concerns including apprehension about being excluded from population screening programs and concern that lowrisk result would lead to negative health behaviors [14]. Similar concerns were not consistently identified in this review. Although some studies reported that heightened risk perception remained even after a low-risk or when no pathogenic variant was identified [10-12, 18], there was also evidence of individuals seeing reduced access to treatment (due to low-risk) as a form of discrimination rather than an opportunity to avoid unnecessary interventions [19]. Another study found no negative health behavior for those at low-risk [13]. Together these highlight the significant influence of perceived risk on screening and treatment decisions. Risk perception is often shaped by lived experiences such as previous diagnosis or family history of cancer rather than genomic results received. If individuals do not accept evidence of reduced risk through genomic testing as a reason to reduce medical interventions, the hoped-for impact of genomic screening on clinical practice could be reduced. Clinicians should be aware of these challenges and ensure appropriate education and counseling is provided when implementing genomic testing.

\section{Study limitations}

Strengths of this review include the use of broad search terms to capture variable nomenclature and inclusion of various test types. This enabled a comprehensive review of genomic testing in cancer. However, our findings should be interpreted in light of the current limitations. Firstly, in the germline setting only three studies assessed panel testing and none assessed WES or WGS, thus there is limited data on the outcomes of such test. Furthermore, the variety of measures used, and the inclusion of non-validated measures limited the comparability of outcomes. Studies were biased towards Caucasian women with high education and socioeconomic status. Only English articles were included, therefore, the results of those within other languages are not captured. Finally, limited longitudinal data limited our ability to assess the long-term impact of genomic testing.

Acknowledgements TY is supported by the National Health and Medical Research Council (NHMRC) and National Breast Cancer Foundation postgraduate scholarship (ID 1133049), and the Translational Cancer Research Institute PhD Top-up Scholarship. BM is supported by an NHMRC Senior Research Fellowship Level B (ID 1078523). MB is supported by the Cancer Institute NSW Early Career Fellowship (ID MB00352).

\section{Compliance with ethical standards}

Conflict of interest KT and BM have had remunerated consultant roles with respect to a study unrelated to this article with the company AstraZeneca. TY, AW, and MB have not conflict of interest to declare.

\section{References}

1. Ringwald J, Wochnowski C, Bosse K, Giel KE, Schaffeler N, Zipfel S, et al. Psychological Distress, Anxiety, and Depression of Cancer-Affected BRCA1/2 Mutation Carriers: a Systematic Review. J Genet Couns. 2016;25:880-91.

2. Heshka JT, Palleschi C, Howley H, Wilson B, Wells PS. A systematic review of perceived risks, psychological and behavioral impacts of genetic testing. Genet. Med.. 2008;10:19-32.

3. Dieng M, Watts CG, Kasparian NA, Morton RL, Mann GJ, Cust AE. Improving subjective perception of personal cancer risk: systematic review and meta-analysis of educational interventions for people with cancer or at high risk of cancer. Psychooncology. 2014;23:613-25.

4. Hatcher MB, Fallowfield L, A'Hern R. The psychosocial impact of bilateral prophylactic mastectomy: prospective study using questionnaires and semistructured interviews. BMJ. 2001;322:76.

5. Moher D, Liberati A, Tetzlaff J, Altman DG. Preferred Reporting Items for Systematic Reviews and Meta-Analyses: The PRISMA Statement. PLoS Med. 2009;6:e1000097.

6. NHGRI, International Consortium Completes Human Genome Project. 2003.

7. Kmet, L., R. Lee, and L. Cook, Standard Quality Assessment Criteria for Evaluating Primary Research Papers from a Variety of Fields. 2004: Edmonton: Alberta Heritage Foundation for Medical Research. 
8. Bradbury AR, Patrick-Miller LJ, Egleston BL, DiGiovanni L, Brower J, Harris D, et al. Patient feedback and early outcome data with a novel tiered-binned model for multiplex breast cancer susceptibility testing. Genet. Med.. 2016;18:25-33.

9. Lumish HS, Steinfeld H, Koval C, Russo D, Levinson E, Wynn J, et al. Impact of Panel Gene Testing for Hereditary Breast and Ovarian Cancer on Patients. J Genet Couns. 2017;1-14.

10. Sie AS, Prins JB, van Zelst-Stams WAG, Veltman JA, Feenstra I, Hoogerbrugge N. Patient experiences with gene panels based on exome sequencing in clinical diagnostics: High acceptance and low distress. Clin. Genet.. 2015;87:319-26.

11. Bancroft E,K, Castro E, Ardern-Jones A, Moynihan C, Page E, Taylor N, et al. "It's all very well reading the letters in the genome, but it's a long way to being able to write": Men's interpretations of undergoing genetic profiling to determine future risk of prostate cancer. Fam. Cancer. 2014;13:625-35.

12. Bancroft EK, Castro E, Bancroft GA, Ardern-Jones A, Moynihan $\mathrm{C}$, Page E, et al. The psychological impact of undergoing geneticrisk profiling in men with a family history of prostate cancer. Psychooncology. 2015;24:1492-9.

13. Smit AK, Espinoza D, Newson AJ, Morton RL, Fenton G, Freeman L, et al. A Pilot Randomized Controlled Trial of the Feasibility, Acceptability, and Impact of Giving Information on Personalized Genomic Risk of Melanoma to the Public. Cancer Epidemiol Biomarkers Prev. 2017;26:212-21.

14. Smit AK, Keogh LA, Newson AJ, Butow PN, Dunlop K, Morton $\mathrm{RL}$, et al. Does personalized melanoma genomic risk information trigger conversations about skin cancer prevention and skin examination with family, friends and health professionals? Br. J. Dermatol.. 2017;177:779-90.

15. Young MA, Forrest LE, Rasmussen VM, James P, Mitchell G, Sawyer SD, et al. Making Sense of SNPs: Women's Understanding and Experiences of Receiving a Personalized Profile of Their Breast Cancer Risks. J Genet Couns. 2018;27:702-08.

16. Diseati L, Scheinfeldt LB, Kasper RS, Zhaoyang R, Gharani N, Schmidlen TJ, et al. Common genetic risk for melanoma encourages preventive behavior change. Pers. Med. 2015;5:36-49.

17. Graves KD, Leventhal KG, Nusbaum R, Salehizadeh Y, Hooker $\mathrm{GW}$, Peshkin BN, et al. Behavioral and psychosocial responses to genomic testing for colorectal cancer risk. Genomics. 2013;102:123-30.

18. Nusbaum R, Leventhal KG, Hooker GW, Peshkin BN, Butrick M, Salehizadeh Y, et al. Translational genomic research: Protocol development and initial outcomes following SNP testing for colon cancer risk. Transl. Behav. Med.. 2013;3:17-29.

19. Bombard Y, Rozmovits L, Trudeau M, Leighl NB, Deal K, Marshall DA. Access to personalized medicine: Factors influencing the use and value of gene expression profiling in breast cancer treatment. Curr Oncol. 2014;21:426-33.

20. Bombard Y, Rozmovits L, Trudeau ME, Leighl NB, Deal K, Marshall DA. Patients' perceptions of gene expression profiling in breast cancer treatment decisions. Curr Oncol. 2014;21:e203-e211.

21. Lipkus I, Vadaparampil S, Jacobsen P, Miree C. Knowledge about genomic recurrence risk testing among breast cancer survivors. J. Cancer Educ.. 2011;26:664-9.
22. Lo SS, Mumby PB, Norton J, Rychlik K, Smerage J, Kash J, et al. Prospective multicenter study of the impact of the 21-gene recurrence score assay on medical oncologist and patient adjuvant breast cancer treatment selection. J. Clin. Oncol.. 2010;28:1671-6.

23. Miller F, Hayeems R, Bytautas J, Bedard P, Ernst S, Hirte H, et al. Testing personalized medicine: patient and physician expectations of next-generation genomic sequencing in late-stage cancer care. Eur. J. Hum. Genet.. 2014;22:391-5.

24. Pellegrini I, Rapti M, Extra JM, Petri-Cal A, Apostolidis T, Ferrero JM, et al. Tailored chemotherapy based on tumour gene expression analysis: breast cancer patients' misinterpretations and positive attitudes. Eur. J. Cancer Care (Engl.). 2012;21:242-50.

25. Retel V, Groothuis-Oudshoorn C, Aaronson N, Brewer N, Rutgers $\mathrm{E}$, van $\mathrm{H}$, et al. Association between genomic recurrence risk and well-being among breast cancer patients. BMC Cancer. 2013;13:295.

26. Richman AR, Tzeng JP, Carey LA, Retel VP, Brewer NT. Knowledge of genomic testing among early-stage breast cancer patients. Psychooncology. 2011;20:28-35.

27. Seror V, Marino P, Bertucci F, Mancini J, Extra JM, Ferrero JM, et al. Breast cancer patients' views on the use of genomic testing to guide decisions about their postoperative chemotherapy. Public Health Genomics. 2013;16:110-17.

28. Sulayman N, Spellman E, Graves KD, Peshkin BN, Isaacs C, Schwartz MD, et al. Psychosocial and Quality of Life in Women Receiving the 21-Gene Recurrence Score Assay: The Impact of Decision Style in Women with Intermediate RS. J. Cancer Epidemiol.. 2012;2012:728290.

29. Tzeng JP, Mayer D, Richman AR, Lipkus I, Han PK, Valle CG, et al. Women's experiences with genomic testing for breast cancer recurrence risk. Cancer. 2010;116:1992-2000.

30. Cella D, Hughes C, Peterman A, Chang CH, Peshkin BN, Schwartz MD, et al. A brief assessment of concerns associated with genetic testing for cancer: the Multidimensional Impact of Cancer Risk Assessment (MICRA) questionnaire. Health Psychol. 2002;21:564-72.

31. Kasparian NA, Wakefield CE, Meiser B. Assessment of psychosocial outcomes in genetic counseling research: an overview of available measurement scales. J Genet Couns. 2007;16:693-712.

32. Horowitz M, Wilner N, Alvarez W. Impact of Event Scale: a measure of subjective stress. Psychosomatic medicine. 1979;41:209-18.

33. Wiseman M, Dancyger C, Michie S. Communicating genetic risk information within families: a review. Fam. Cancer. 2010;9: 691-703.

34. Pashayan N, Morris S, Gilbert FJ, Pharoah PP. Cost-effectiveness and benefit-to-harm ratio of risk-stratified screening for breast cancer: A life-table model. JAMA Oncol. 2018. https://doi.org/10. 1001/jamaoncol.2018.1901.

35. Henneman L, Timmermans DR, Bouwman CM, Cornel MC, Meijers-Heijboer H. 'A low risk is still a risk': exploring women's attitudes towards genetic testing for breast cancer susceptibility in order to target disease prevention. Public Health Genomics. 2011;14:238-47. 